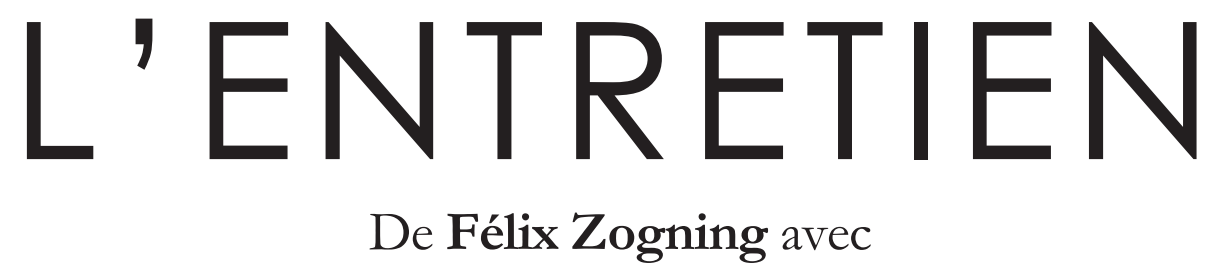

\title{
Anthony Rjeily \\ Associé et leader national en transformation numérique et innovation chez EY
}

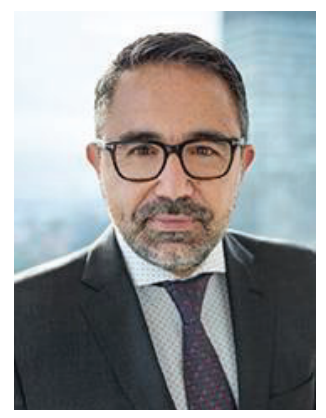

Photo : EY Photography

Depuis la dernière crise financière internationale de 2008, où les banques ont sévèrement été critiquées, le secteur de la technologie financière (ou fintech) n'a cessé de se développer et d'innover, entretenant avec le secteur bancaire des relations à la fois aux allures de compétition et de collaboration. Même si l'utilisation de la technologie en soutien aux services financiers n'est pas récente, son essor au cours de la dernière décennie, notamment son adoption par les institutions financières, semble avoir accéléré le virage numérique et renforcé ses capacités à affronter une nouvelle crise. Les banques constituent l'une des industries dont l'activité est le moins affectée par les restrictions qu'impose la pandémie de COVID-19.

Anthony Rjeily est associé chez Ernst \& Young (EY) et leader national de ladite firme en transformation numérique et innovation. Il cumule plus de 25 années d'expérience et accompagne des entreprises de différents secteurs dans leurs projets de croissance par le biais de l'engagement numérique. Il nous explique dans cet entretien en quoi la fintech a rendu les banques commerciales plus agiles et leur a permis de rapidement s'adapter à l'avènement de la pandémie.

... Vous êtes leader du groupe EY au Canada en transformation numérique et innovation. Votre firme a le mandat d'accompagner des entreprises dans divers secteurs. Quel est le rôle d'un leader en transformation numérique dans une telle firme?

Anthony Rjeily. La transformation numérique et innovation fait partie de la pratique consultation chez EY. Notre firme participe dans quatre secteurs"d'affaires : comptabilité et vérification, fiscalité, redressement d'entreprises et consultation. La pratique que je mène est du côté de la consultation. Nous avons bâti notre pratique de transformation numérique et d'innovation pour aider nos clients à faire face aux nouvelles tendances dans le monde 
des affaires. Comme on le sait, la transformation numérique, les nouvelles technologies et les données de masse (big data) ont eu un grand impact ces dernières années chez toutes les organisations, dans tous les secteurs d'activité, et ça va continuer. Nous avons imaginé des solutions pour aider nos clients à livrer leur stratégie de transformation numérique. En d'autres termes, comment la transformation numérique peut aider les entreprises à livrer leur plan d'affaires à travers la fonction technologique.

La transformation numérique, c'est très important de la définir parce que je pense que, si vous parlez à plusieurs personnes dans le marché, vous allez obtenir une définition différente et vaste. C'est quoi, la transformation numérique? Nous avons passé beaucoup de temps avec nos clients dans différentes industries pour en établir une définition. Et pour la résumer, c'est en trois volets.

Le premier volet est ce qu'on appelle l'interaction et l'engagement avec les clients. Chaque organisation doit interagir avec ses clients, que ce soit dans les entreprises à consommateur en ligne (business-toconsumer) ou le commerce entre entreprises (businessto-business). Alors, quelle est l'expérience que cette entreprise doit donner à ses clients en matière d'interaction pour l'achat de produits et services ou pour le côté soutien et services? Le côté interaction en général doit vraiment être transformé. Quelques exemples : nous avons le cas des banques qui proposent une nouvelle expérience numérique, soit pour l'ouverture d'un compte ou pour l'approbation de crédit. Ces expériences avec leurs clients ont beaucoup été touchées par la transformation numérique jusqu'à maintenant. En plus, il y a un autre aspect très important, qui est la transformation numérique au sein de l'entreprise et des employés. Aujourd'hui, vous pouvez ouvrir un compte de banque sur le site web d'une banque $\mathrm{X}$, mais l'orchestration de tous les processus et la façon dont les gens travaillent dans la banque et dont ils gèrent les données doivent être transformées également pour soutenir cette expérience client. Donc, la transformation numérique de l'entreprise et l'expérience employé sont très importantes.

Le deuxième volet, c'est de regarder à l'interne comment transformer les processus, l'information, les technologies et la façon de travailler pour être aligné avec la façon dont l'entreprise veut s'engager avec ses clients. Et le troisième volet, c'est le côté innovation: penser à de nouveaux modèles d'affaires, produits et services, à un écosystème de différents partenaires qu'une entreprise doit avoir. Ça fait partie également de la définition de la transformation numérique.

Chez EY, nous avons établi ces trois dimensions de la transformation numérique et, sous chacune des dimensions, nous avons développé des solutions pour aider les clients à faire face à cette transformation essentielle.

Si on prend le cas particulier des institutions financières, on note qu'elles connaissent une implantation grandissante de la technologie financière, des innovations financières. On l'a vu avec les robots conseillers en gestion de placements. On le voit également dans tous les produits d'assurances ainsi que dans la numérisation des services et produits bancaires. Qu'est-ce que les institutions financières tirent comme avantages dans cette implantation grandissante de la fintech?

A. R. La dynamique entre la fintech et les grandes institutions financières est quand même assez intéressante. Depuis les trois à quatre dernières années, cette dimension a vraiment évolué. Je vous dirais que les avantages pour les institutions financières ont commencé depuis trois ou quatre ans. Lorsque la fintech a commencé à entrer dans le marché, les institutions financières ne lui ont pas vraiment donné beaucoup de chances d'avoir du succès. Mais, très rapidement, je vous dirais dans les 18 à 24 derniers mois, on a vu qu'elles ont commencé avoir pas mal de succès, en plus d'avoir une part de marché intéressante dans certains segments de la population. Donc, les institutions financières l'ont vue comme un compétiteur et se sont dit: on va bâtir nous-mêmes notre technologie et ces expériences-là pour faire face à cette perturbation importante.

Toutefois, elles se sont rendu compte qu'elles n'avaient pas autant d'agilité ou même de culture d'innovation. Et leur perception de l'industrie de la fintech a évolué pour y voir l'opportunité de faire des partenariats ou même des investissements stratégiques. Il y a maintenant plusieurs institutions financières qui ont bâti des centres d'innovation ou d'incubation, où elles amènent des partenaires en fintech pour voir comment elles peuvent potentiellement développer des solutions communes ou même comment prendre leurs solutions et les intégrer au sein des produits et services des institutions financières. D'autres institutions financières ont 
même fait des investissements importants dans certains secteurs de la fintech. On a donc vu des acquisitions se faire.

Je pense que, quand on observe ces deux dynamiques (fintech et institutions financières), il y a beaucoup de complémentarité. Du côté des banques, elles ont manifestement accès à un vaste bassin de clients et le niveau de confiance avec leurs clients est très élevé. D'ailleurs, leur taux de confiance est plus élevé que dans n'importe quelle autre industrie. Elles ont accès à des canaux de distribution assez larges, que ce soit en online ou offline. Par contre, elles sont assises sur des infrastructures technologiques assez vieilles et n'ont pas nécessairement fait beaucoup d'innovations dans le passé.

Si on observe les pour et les contre entre la fintech et les institutions financières, c'est vraiment le miroir inversé. La fintech a accès à l'innovation, est très agile et créative; elle bouge très rapidement. Par contre, elle n'a pas accès à d'autres canaux de distribution à part le numérique. Elle n'a pas encore la confiance élevée de la part des consommateurs ou des entreprises. C'est là que les institutions financières ont vu des avantages à s'associer d'une façon ou d'une autre avec la fintech.

Toutefois, je pense que le modèle d'intégration est encore à trouver. C'est très difficile pour une grande entreprise de bâtir un modèle d'innovation où elle pourrait tirer profit de la fintech sans pour autant la tuer, une fois qu'elle entre dans l'organisation. À cause de la taille des institutions financières par rapport à la fintech, c'est difficile de protéger cette dernière, de s'assurer qu'elle livre la valeur envisagée. C'est là que nous intervenons. Nous avons de l'expérience pour aider les entreprises à identifier les secteurs d'activité où certains secteurs de la fintech pourraient apporter de la valeur. Mais, encore une fois, dès que c'est identifié, il faut bâtir un modèle d'intégration pour aller chercher la valeur qui est là, mais qui est parfois difficile à faire ressortir.

Dans les pays en développement, il est clair que la technologie financière améliore grandement l'inclusion financière. Dans des pays de l'OCDE ou du G7 comme le Canada, est-ce qu'on peut penser que la contribution de cette technologie financière est tout aussi importante en matière d'inclusion financière?

A. R. Je pense que oui. Il y a une inclusion financière sur deux plans : l'inclusion financière dans le sens de promouvoir des produits et services qui n'étaient pas nécessairement disponibles à certains segments de la population. Malgré le fait que c'est très évident dans les pays en développement, on voit qu'il y a aussi dans les pays développés des segments de la population ou même du côté des PME qui n’ont pas nécessairement accès aux produits et services dont ils ont besoin auprès des institutions financières. La fintech a amené dans le marché certaines solutions qui ont augmenté l'inclusion de ces segments du marché, même dans les pays développés.

Alors, l'autre côté de la médaille, c'est aussi le développement de la main-d'œuvre. Je pense qu'au Canada, on est un leader en innovation de la fintech. Dans le passé, avec le gros boom Internet, on voyait beaucoup de notre main-d'œuvre exportée aux ÉtatsUnis, à la Silicon Valley. Au cours des cinq dernières années, depuis la venue de la fintech et de l'innovation en général, avec l'intelligence artificielle ou même des produits et services connexes des institutions financières, on constate que le talent reste au Canada. Donc, je pense qu'il y a de l'inclusion à l'échelle du marché, mais aussi de la main-d'œuvre puisqu'on est en mesure de garder ce talent au Canada, de le développer et de le promouvoir pour permettre aux entreprises canadiennes d'accéder à un marché global parce que les racines sont numériques et la fintech n'a pas de frontières.

Parlons de la pandémie de COVID-19. Les effets sont néfastes et quasiment dévastateurs pour plusieurs secteurs d'activité. Comment se comportent les institutions financières face à ce défi?

A. R. Oui, c'est un aspect très important. Je dirais que la pandémie a significativement accéléré la transformation numérique pour toutes les entreprises, que ce soit dans le domaine financier ou dans n'importe quelle autre industrie. Les plans de transformation numérique qui s'étalaient sur trois ou cinq ans ont été maintenant réduits à quelques mois. La raison, c'est évidemment que les gens ne peuvent plus faire affaire avec les institutions financières de la façon traditionnelle : rencontrer leur gestionnaire de portefeuille, se rendre dans une succursale pour faire leurs transactions, demander un prêt hypothécaire en personne, etc. Il y a eu énormément d'investissements du côté numérique dans les services financiers, mais cela a rapidement accéléré depuis les 10 derniers mois. Il y a beaucoup d'investissements qui ont été réalisés au sein 
des organisations pour s'assurer que les produits et services pour les clients soient complètement numériques.

Maintenant, cela amène aussi des dimensions assez intéressantes quant au modèle d'affaires. Plusieurs banques demandent à leurs employés de travailler de la maison, sauf pour les services essentiels en succursale. Et même là, dans certaines succursales, le personnel est beaucoup plus réduit, et ce, pour deux raisons : les gestionnaires veulent que les employés travaillent à la maison, mais, en plus, les clients ne viennent plus beaucoup en succursale. Ils doivent donc absolument faire un pivot pour offrir leurs services numériquement, mais aussi investir énormément dans leurs employés sur la dimension virtuelle afin qu'ils puissent travailler à distance. L'expérience employé a dû être réinventée par le biais du numérique pour faire face à la pandémie.

La troisième chose que je vous dirais, c'est que la structure des coûts opérationnels dans les succursales constitue en moyenne $65 \%$ des coûts fixes d'une institution financière. Qu'est-ce qu'on fait avec ça? Je pense que la pandémie offre une opportunité de réduire ces coûts en optimisant le nombre de succursales ou en réinventant l'expérience client dans ces succursales pour le futur. Donc, on n'aura plus besoin d'avoir des succursales qui sont exactement les mêmes. On a vraiment l'occasion d'étudier quels clients viennent en succursale, leurs besoins et leurs préférences, puis d'offrir des expériences très spécifiques par succursale pour avoir des modèles différents de distribution. C'est une opportunité énorme pour les banques.

Avant la pandémie, plusieurs banques avaient déjà grandement investi dans les innovations financières. Et, comme vous le dites, cela s'est accéléré pendant la pandémie. Est-ce que les banques ou, plus largement, les institutions financières qui étaient déjà bien avancées dans ces innovations ont pu faire la différence? Est-ce que le fait d'avoir été bien équipé, même avant la pandémie, leur a permis de passer plus facilement à travers la crise? Si oui, qu'est-ce qui pourrait l'expliquer?

A. R. Oui, il y a une différence sur deux plans. La première différence est que l'expérience client est quand même assez diverse parmi les institutions financières. Celles qui ont vraiment investi dans les innovations financières ont eu une longueur d'avance au début de la pandémie. Je pense qu'on a continué à voir cela durant la pandémie. C'était important parce que les clients ont subitement arrêté d'aller en succursale. Ce n'était pas une transition de plusieurs mois où les institutions financières avaient le temps de développer une expérience. Ça s'est fait très rapidement et on a pu voir une différence entre les institutions financières qui avaient investi et mis beaucoup l'accent sur l'expérience client, et les autres. Il y a certes eu du rattrapage chez les autres, mais, en même temps, on voit quand même qu'il reste une bonne différence.

La seconde différence, c'est que la pénétration numérique était assez faible pour les institutions financières, même si les clients utilisaient de plus en plus leurs canaux numériques pour avoir accès à leurs produits et services. Donc, les investissements faits au cours des trois à cinq dernières années dans les institutions financières étaient beaucoup plus axés sur le front-end. Vous pouviez ouvrir un compte de banque ou demander un crédit en ligne, mais ce qui se faisait après la soumission de votre demande, c'était toujours le même processus, souvent très manuel et qui utilisait beaucoup de paperasse. Le modèle opérationnel, les processus, la façon de travailler: tout était encore très manuel. Les institutions financières qui avaient investi avant la pandémie dans ces aspects et dans la technologie ont vu qu'elles pouvaient faire leur boulot beaucoup plus rapidement et de façon beaucoup plus efficiente.

La façon dont je catégoriserais les expériences pré-COVID de plusieurs institutions financières, ce sont des expériences numériques qui n’étaient pas nécessairement optimisées ou profitables parce que le service d'accueil (front-end) était considéré, mais pas les tâches de traitement (back-end). Or, pour survivre et pouvoir générer les profits, on doit avoir des expériences de bout en bout qui concernent le front-end, mais aussi qui numérisent les processus, les données, la technologie et l'expérience employé. Je pense que c'est là que l'accent sera mis en matière d'investissements numériques pour les prochaines années.

On peut donc penser que l'innovation dans le secteur de la technologie financière est susceptible de s'accélérer encore plus, dans ce monde de plus en plus numérique après la pandémie de COVID-19. Quel sera l'avenir de la fintech après la crise?

A. R. D'abord, les habitudes des clients et des consommateurs ont changé au cours des 10 derniers mois et d'une façon beaucoup plus permanente qu'on pourrait le penser. Je pense que les gens se 
sont habitués à demander des expériences numériques, qui vont surement continuer. Il y a énormément d'industries qui vont devoir continuer l'innovation requise pour optimiser les processus et l'expérience client, et pour continuer à innover en collaboration avec plusieurs joueurs dans leur écosystème, que ce soit la fintech, des centres de recherche et autres. Je pense que l'investissement y est soutenu et va continuer de l'être.

L'autre chose intéressante, c'est qu'on a vu que la longueur des cycles d'innovation durant la pandémie a significativement été écourtée. Souvent, on voyait des produits et services financiers qui pouvaient prendre entre 24 et 36 mois de développement, du design jusqu'à la mise en marché. Ce qu'on a vu depuis le début de la pandémie, c'est qu'ils ont été ramenés à quelques semaines. Donc, je pense que cela va beaucoup réajuster les attentes en matière de durée du cycle d'innovation et de temps du retour sur investissement.

La vague de fintech est issue en grande partie du chaos engendré par la crise financière mondiale de 2008. D'après ce que vous dites, pendant la crise de COVID-19, son implantation s'est largement densifiée. On a plutôt le sentiment que, finalement, les crises alimentent l'innovation.

A. R. Oui, absolument! Je pense que, dans toute crise, il y a des opportunités d'amélioration ou d'innovation pour toutes les industries. Le secteur financier s'est rebâti avec la crise financière de 2008. Mais je pense qu'il y a aussi d'autres dimensions qui ont fait en sorte que l'entrée de la fintech ait pu se faire. L'accès à la technologie est nettement meilleur que ce qu'il en était avant la crise financière. Le coût des technologies a énormément diminué, l'accès aux données est simplifié et leur coût est plus abordable. Je pense aussi que les attentes des consommateurs ont beaucoup évolué, qu'il y a plusieurs vecteurs qui ont produit cette innovation généralisée.

Je crois que la fintech a tiré avantage de ces dimensions parce qu'elle avait accès au capital assez rapidement. Il y avait beaucoup d'argent disponible dans le marché, le talent était là et les gens voulaient des solutions innovatrices. Le niveau de confiance envers les institutions financières était quand même très bas après la crise économique. Donc, les gens étaient beaucoup plus ouverts à faire affaire avec des institutions non financières pour leurs services financiers. Et cela a créé toute la dynamique qu'on voit aujourd'hui.

La fintech a le vent en poupe et a réussi à établir des partenariats avec des banques. Jusqu'ici, cela a bien fonctionné. Mais il y a un contraste. En ce qui a trait au financement de ces entreprises de fintech, elles ne se portent pas toutes bien en temps de pandémie. Il y en a qui ont connu des moments très difficiles. De façon plus large, on peut noter que leur financement est en baisse. Au deuxième trimestre 2020, ces entreprises ont globalement levé un peu moins de 6,5 G\$, ce qui est tout de même assez bas par rapport à ce qu'elles ont eu l'habitude de lever comme fonds dans les dernières années. Alors, d'un côté, on sait qu'il y a de plus en plus de demandes pour la fintech, mais, de l'autre, on se demande si elles vont pouvoir livrer la marchandise, compte tenu du fait que leur financement est en baisse. Comment cela peut-il s'expliquer?

A. R. C'est sûr que la pandémie a rendu les investisseurs un peu plus prudents. En même temps, je pense que le niveau de maturité de la fintech a évolué au cours des dernières années, de sorte qu'il y a une certaine consolidation ou concentration des investissements qui commence à se faire dans différents secteurs de la fintech. On voit aussi beaucoup d'entreprises de fintech qui ont évolué, maturé pour arriver à une certaine taille et ont elles-mêmes développé des produits et services connexes. Comme n'importe quel cycle d'innovation, il y a énormément de fragmentation au début. L'argent est distribué à travers plusieurs idées très tôt dans le cycle de financement. Une fois qu'il y a certains degrés de maturité dans l'industrie, une certaine prudence ou concentration des investissements s'installe. Il y a moins d'investissements en nombre, mais les montants investis sont plus importants. Ce sont ces dimensions qui existent présentement dans le marché, ce qui fait en sorte qu'il y a moins d'opportunités pour les investisseurs parce qu'il y a une certaine maturité du secteur et que les investissements affichent différentes dynamiques.

En conclusion, quel autre aspect de l'industrie soubaitezvous aborder?

A. R. Un autre point intéressant en innovations financières et en fintech, ce sont les GAFAM (ou Big $T e c b)$, les géants du Web, qui sont en revanche déjà dans le domaine financier. Amazon est une banque. Uber l'est aussi. Ils ont des services financiers, font du financement, émettent des cartes de crédit, créent des comptes bancaires. Les banques s'inquiètent avec 
raison parce qu'elles craignent que ces joueurs compétitionnent dans leur industrie. Or, ils sont déjà là. Ils ont une dynamique très différente des entreprises de fintech parce qu'ils ont accès à énormément d'informations de leurs clients et ont la confiance des consommateurs. Ils peuvent donc facilement développer des produits et services numériques pour compétitionner et prendre une grande part de marché. Je pense que c'est là le plus grand risque qui existe chez les institutions financières plus traditionnelles.

Anthony Rjeily, merci d'avoir accepté notre invitation. Je vous remercie également pour ces explications fort éclairantes.

A. R. Ça m'a fait plaisir. Merci beaucoup de m'avoir donné l'opportunité de discuter de ces questions. ..• 